\title{
Variación Retroesofágica del Arco Aórtico
}

\author{
Retroesophageal Variation of the Aortic Arch
}

"Rubén Daniel Algieri; ** Martín Javier Mazzoglio y Nabar \& *María Soledad Ferrante

\begin{abstract}
ALGIERI, R. D.; MAZZOGLIO Y NABAR, M. J. \& FERRANTE, M. S. Variación retroesofágica del arco aórtico. Int. J. Morphol., 26(2):337-343, 2008.
\end{abstract}

RESUMEN: La literatura clásica no menciona la frecuencia de variaciones vasculares torácicas, pero estas anormalidades son frecuentes, pudiendo existir sin producir síntomas ni requerir tratamiento. El arco aórtico retroesofágico puede presentarse a la derecha (AARED) o izquierda de la tráquea y esófago y causar síntomas de compresión mediante un anillo vascular parcial o completo. Puede acompañarse de anormalidades de la arteria subclavia. En 1735, Hunauld reporta el primer caso de arteria subclavia aberrante; más tarde, Hommel publica un doble arco aórtico y en 1794 Bayford describe la "disfagia lusoria esofágica obstructiva". Descripción anatómica y correlato clínico quirúrgico de una variación del arco aórtico como un AARED. Se realizó la disección cervicotorácica de un cadáver masculino adulto con AARED, hallado durante el período 03/1998-03/2006, en 95 disecciones. En el AARED, la aorta ascendente transcurre sobre el lado derecho del esófago y la tráquea, cruzando sobre el bronquio principal derecho, el arco aórtico cruza posterior al esófago y desciende sobre el lado izquierdo. Con el ligamento arterioso conforman un anillo vascular que envuelve tráquea y esófago. El sujeto disecado presenta un AARED con anillo vascular completo y una arteria subclavia derecha retroesofágica. Estas variaciones pueden ser reconocidas por imágenes (esofagograma, angiografía o tomografía computarizada con o sin reconstrucción en 3D) ante la presencia de un síndrome por compresión esófago-traqueal. Resulta importante el conocimiento de estas anomalías para el cirujano en abordajes mediastinales por vía cervical o en la urgencia del trauma de tórax.

PALABRAS CLAVE: Variaciones del arco aórtico; Arco aórtico retroesofágico; Anillo vascular; Arteria subclavia retroesofágica.

\section{INTRODUCCIÓN}

La literatura clásica (Testut \& Latarjet, 1981; Williams \& Warwick, 1992; Rouvière \& Delmas, 1999; Latarjet \& Ruiz-Liard, 2005) no indica la frecuencia de la variabilidad morfológica de los vasos en el tórax, pero las anormalidades vasculares en esta región anatómica son más comunes de lo que generalmente se cree, pudiendo existir sin producir síntomas ni requerir tratamiento (Blumenthal \& Ravitch, 1957).

Un gran número de variaciones del arco aórtico y sus vasos han sido descritas en la literatura científica. Las distintas publicaciones utilizaron diversos métodos de estudio como autopsia, disecciones, estudios por imágenes radiográficas, angiografías, resonancias magnéticas y hasta las actuales reconstrucciones en 3D.
En 1735, Hunauld reporta el primer caso de arteria subclavia aberrante (Almear-García et al., 2002; Blumenthal \& Ravitch). Dos años más tarde, Hommel publica la imagen de un doble arco aórtico sobre la base de sus estudios por autopsia de cadáveres humanos. En el 1794, Bayford describe el término de "disfagia lusoria esofágica obstructiva" a causa de la arteria subclavia retroesofágica (Almear-García et al.; Terracol, 1938). Posteriormente, otros autores extendieron la denominación latina lusoria a toda disfagia producida por las diversas anomalías del arco aórtico.

«Lusoria» proviene del latín lusorius que significa relativo al juego, divertimento; el término se conecta con el verbo ludo. Fue en este sentido que Bayford buscó en el latín el adjetivo lusoria, queriendo significar un divertimento

\footnotetext{
* Médico Especialista en Cirugía General. Prof. Adjunto de Anatomía e Histología, Universidad de Morón. Jefe Trabajos Prácticos de Anatomía, Facultad de Medicina, Universidad de Buenos Aires. Argentina.

* Médico Ayudante de $1^{\circ}$ de Anatomía, Facultad de Medicina, Universidad de Buenos Aires. Ayudante de $2^{\circ}$ de Farmacología, Facultad de Medicina, Universidad de Buenos Aires, Argentina.

**** Médica Especialista en Cirugía General. Ayudante de Anatomía e Histología, Universidad de Morón, Argentina.
} 
de la naturaleza que se desvíe del patrón normal (lusus naturae, juego natural).

Con el correr del siglo XIX y durante todo el transcurso del siglo XX se reportaron diversos casos de variaciones del arco aórtico y de sus ramas (principalmente anormalidades de la arteria subclavia) (Tabla I). Asimismo, se determinó en cada trabajo, la incidencia de cada anormalidad sobre la base de la población estudiada y se trazaron teorías con sustento embriológico para explicar estos hallazgos. La mayor prevalencia de anormalidades es referente a la arteria subclavia en ubicación retroesofágica, pudiendo esta misma estar secundada por otras anormalidades. Variaciones vasculares del arco aórtico no son muy frecuentes y su combinación con otras anormalidades vasculares son tomadas como hallazgos.

El embrión, al ingresar a la cuarta semana de desarrollo, posee un corazón tubular en reemplazo de los dos tubos cardíacos primitivos presentes en el disco embrionario trilaminar. La pared de este corazón tubular, se encuentra formada por las mismas capas del corazón adulto y pre-

Tabla I. Porcentaje de casos de anormalidades vasculares del arco aórtico y sus ramas reportadas en humanos según autor y material y método utilizado.

\begin{tabular}{|c|c|c|}
\hline Publicación & $\%$ casos & Método \\
\hline Quain, 1844 & 4 & Autopsia \\
\hline Goldbloom, 1922 & 12 & Autopsia \\
\hline Cairne, 1925 & 1,5 & Disección \\
\hline Adachi, 1928 & 0,2 & Disección \\
\hline Williams, 1932 & 2,5 & Disección \\
\hline Groos, 1946 & 0,4 & disección \\
\hline Evans, 1950 & 3,6 & autopsia en Sme. Down \\
\hline Liechty, 1957 & 1,3 & disección \\
\hline Roszel, 1966 & 0,8 & disección \\
\hline Hastreiter, 1966 & 3,1 & $\mathrm{Rx}$ \\
\hline Goldstein, 1965 & 37 & Rx Sme. Down \\
\hline Bidet, 1977 & 12 & autopsia en Tetralogia de Fallot \\
\hline Balaji, 1982 & 1,1 & población \\
\hline Lippert, 1985 & 1 & población \\
\hline Bergman, 1987 & 1,2 & población \\
\hline Proto, 1988 & 0,9 & Rx contrastada \\
\hline Nakajima, 1990 & 6 & imágenes en Tetralogía de Fallot \\
\hline Harrison, 1994 & 0,5 & población \\
\hline Biull, 1994 & 1,2 & angiogramas \\
\hline Turkenburg, 1994 & 1,2 & $\mathrm{RM}$ \\
\hline Azakie, 1998 & 0,75 & población \\
\hline Wu, 2001 & 0,008 & población \\
\hline Almear-García, 2002 & 0,004 & disección \\
\hline
\end{tabular}

senta 4 dilataciones sucesivas que se denominan: bulbo cardíaco, ventrículo, atrio y seno venoso. En esta semana, el corazón se pliega adaptándose al espacio pericárdico, resultado en la disposición definitiva de sus cavidades. La sangre sale del ventrículo común del corazón hacia una raíz aórtica ventral, desde donde se distribuye por 6 arcos aórticos bilaterales que desembocan en el par de arterias aortas dorsales, en donde la sangre entra en la circulación sistémica regular.

En la parte distal del bulbo se forman 4 cojinetes endocárdicos, dos de ellos se fusionan para constituir un tabique bulbar distal que separa el orificio pulmonar ventral del orificio aórtico dorsal. En el interior del tronco arterioso se proyectan dos engrosamientos endocárdicos en forma de cresta, que en forma espiralada terminan dividiendo a éste en los dos tractos de salida del corazón. Esta diferenciación de las arterias aorta y pulmonar lleva consigo la incorporación y obliteración de distintos segmentos de los arcos aórticos.

Así, la parte caudal del saco aórtico se incorpora al tronco pulmonar y el extremo craneal se divide en ramas derecha, futuro tronco braquiocefálico; e izquierda, que formará la porción ascendente de la aorta y la del arco aórtico entre los orificios del tronco braquiocefálico y la arteria carótida común izquierda. El IV arco aórtico izquierdo forma el arco aórtico entre este último orificio y el de la arteria subclavia izquierda. Caudal a la $7^{\circ}$ arteria intersegmentaria (que en ambos lados originaran a las arterias subclavias) la aorta dorsal derecha desaparece y la izquierda forma la parte descendente del arco aórtico. A partir de ese punto, las aortas dorsales embrionarias derecha e izquierda persisten formando juntas la aorta torácica y abdominal definitivas. Las porciones iniciales de ambas arterias carótidas comunes derivan de la raíz aórtica ventral entre los arcos aórticos III y IV. En cambio, las arterias coronarias se forman in situ y conectan secundariamente con la aorta (Carlson, 2000; Williams \& Warwick).

El arco aórtico retroesofágico (AARE), en el cual el arco aórtico cruza la línea mediana posterior al esófago hacia el lado contralateral, es una forma rara de anormalidad vascular (Figs. 2 y 3 ). Puede presentarse como AARE derecho (AARED) o AARE izquierdo (AAERI) y puede causar síntomas de compresión mediante un anillo 


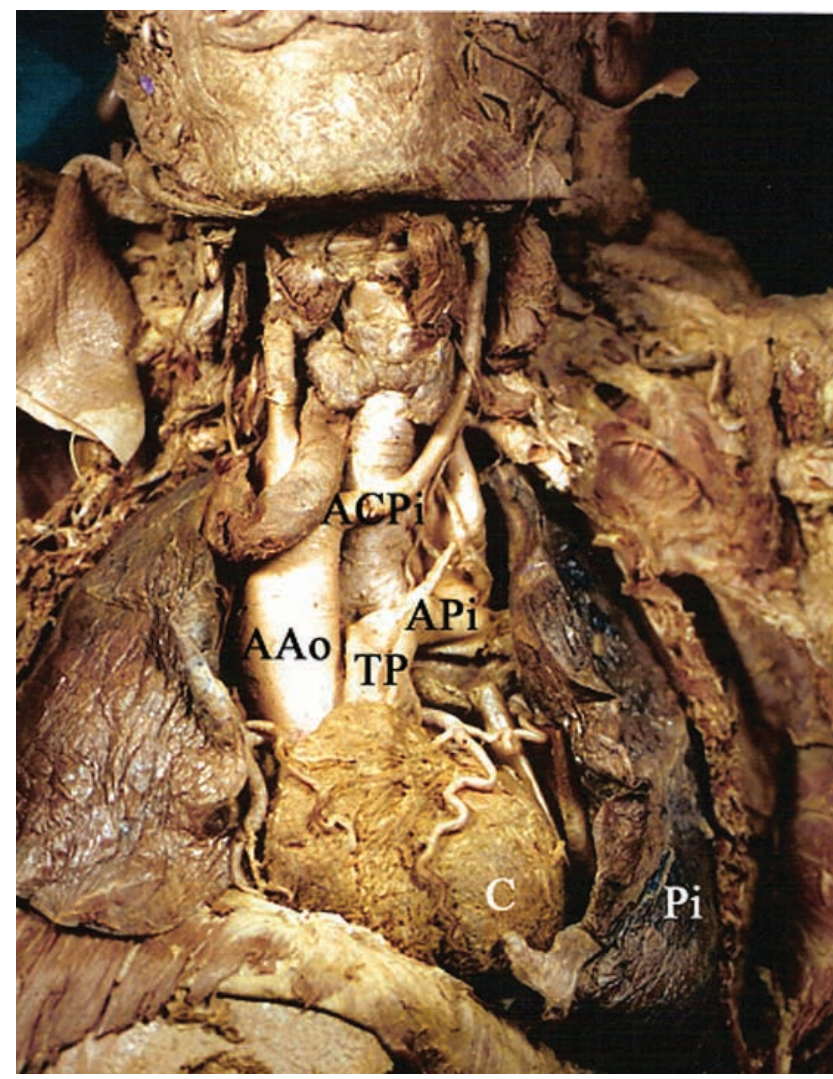

Fig. 1. Vista anterior completa del cadáver. Se pueden apreciar el corazón $(\mathrm{C})$, pulmón izquierdo $(\mathrm{Pi})$, arco aórtico (AAo), tronco arteria pulmonar (TP), arteria carótida común izquierda (ACPi) y arteria pulmonar izquierda (APi).

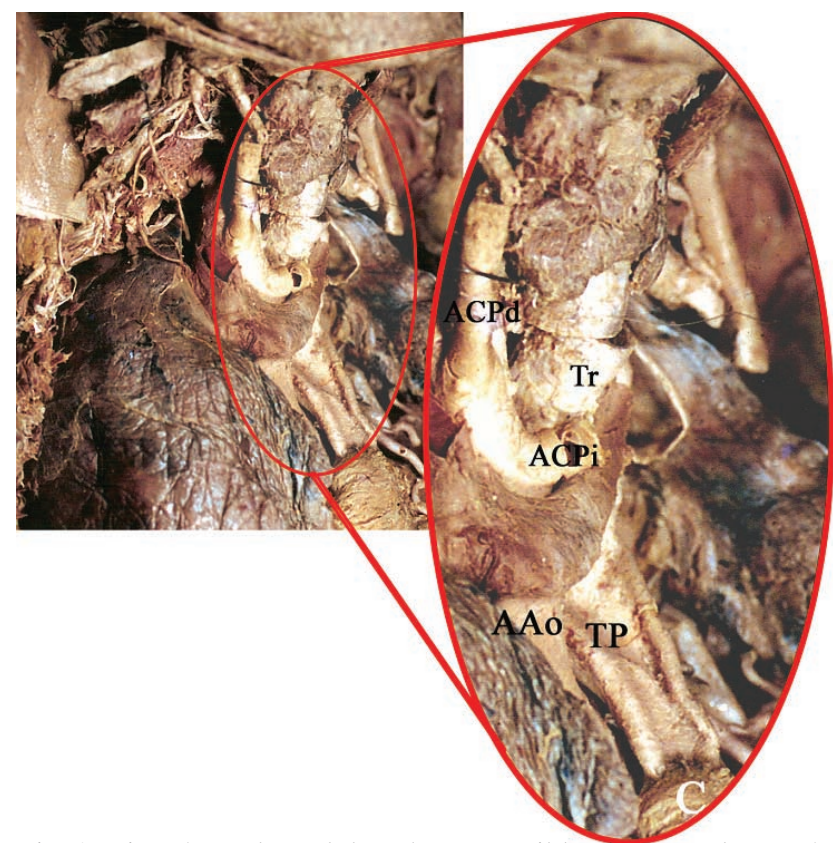

Fig. 2. Vista ánterolateral derecha. Es posible observar el corazón (C), arco aórtico (AAo), tronco de la arteria pulmonar (TP), arterias carótidas comunes derecha (ACPd) e izquierda (ACPi) y la tráquea $(\mathrm{Tr})$. vascular (Arciniegas, 1979). El anillo vascular puede ser parcial o completo. El tipo completo de anillo vascular es formado por un AARE combinado con un ligamento arterioso o canal arterioso manifiesto. Esta forma completa puede causar síntomas de compresión y requiere intervención quirúrgica. Las manifestaciones clínicas serán por compresión traqueo-esofágica de tipo mecánicas.

El objetivo del presente trabajo es describir anatómicamente y realizar un correlato anatomoclínico de la peculiar variación anatómica. Asimismo, focalizar la importancia de esta variación para el conocimiento del cirujano.

\section{MATERIAL Y MÉTODO}

Se realizó la disección cervicotorácica de un cadáver masculino, adulto, formalizado al 10\%, con AARED hallado en el área de Disección de la III Cátedra de Anatomía de la Universidad de Buenos Aires, Argentina, en un total de 95 disecciones llevadas a cabo en el período comprendido entre marzo de 1998 y marzo de 2006. Las diferentes estructuras cervicales y mediastinales fueron identificadas. Puntualizada la variación se estudiaron minuciosamente el arco aórtico, los nervios vagos, los nervios frénicos, y elementos pulmonares, como también la glándula tiroides, la tráquea, los bronquios, troncos venosos, la venas ácigos, la vena hemiácigos, el conducto torácico y la cadena paravertebral, con el fin de detectar malformaciones o patologías posibles en las estructuras mencionadas.

\section{RESULTADOS}

En el AARE derecho, la arteria aorta ascendente transcurre sobre el lado derecho del esófago y la tráquea cruzando sobre el bronquio fuente derecho (Figs. 1, 4 y 5). El arco aórtico sigue su curso con dirección izquierda e inclinado posterior al esófago y descendente sobre el lado izquierdo (Figs. 4 y 5).

La forma de anillo vascular completo se presenta en este caso, por la presencia del ligamento arterioso (Figs. 3, 4 y 5). La arteria subclavia derecha tiene su origen en el arco aórtico, en posición retroesofágica (Figs. 5 y 6).

La carótida primitiva derecha tiene su origen en el arco aórtico y la carótida primitiva izquierda en la arteria aorta ascendente describiendo un trayecto pretraqueal (Figs. $1,3,4,5$ у 6$)$. 

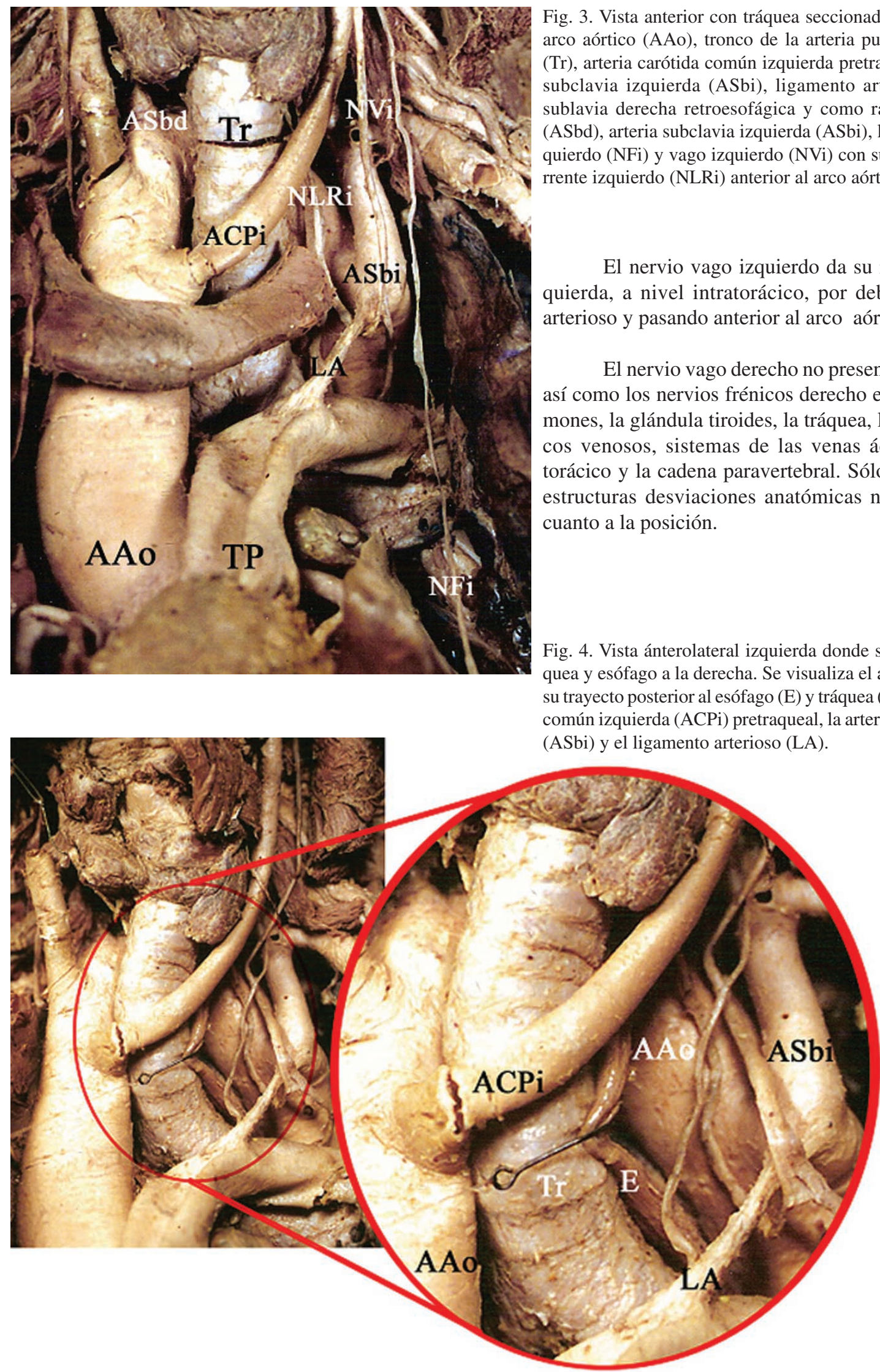

Fig. 3. Vista anterior con tráquea seccionada donde se aprecia el arco aórtico (AAo), tronco de la arteria pulmonar (TP), tráquea (Tr), arteria carótida común izquierda pretraqueal (ACPi), arteria subclavia izquierda (ASbi), ligamento arterioso (LA), arteria sublavia derecha retroesofágica y como rama del arco aórtico (ASbd), arteria subclavia izquierda (ASbi), los nervios frénico izquierdo (NFi) y vago izquierdo (NVi) con su ramo laríngeo recurrente izquierdo (NLRi) anterior al arco aórtico.

El nervio vago izquierdo da su rama recurrente izquierda, a nivel intratorácico, por debajo del ligamento arterioso y pasando anterior al arco aórtico (Figs. 2 y 4).

El nervio vago derecho no presenta particularidades, así como los nervios frénicos derecho e izquierdo, los pulmones, la glándula tiroides, la tráquea, los bronquios, troncos venosos, sistemas de las venas ácigos, el conducto torácico y la cadena paravertebral. Sólo se hallan en estas estructuras desviaciones anatómicas no significativas en cuanto a la posición.

Fig. 4. Vista ánterolateral izquierda donde se han rebatido la tráquea y esófago a la derecha. Se visualiza el arco aórtico (AAo) en su trayecto posterior al esófago (E) y tráquea (Tr), la arteria carótida común izquierda (ACPi) pretraqueal, la arteria subclavia izquierda (ASbi) y el ligamento arterioso (LA). 


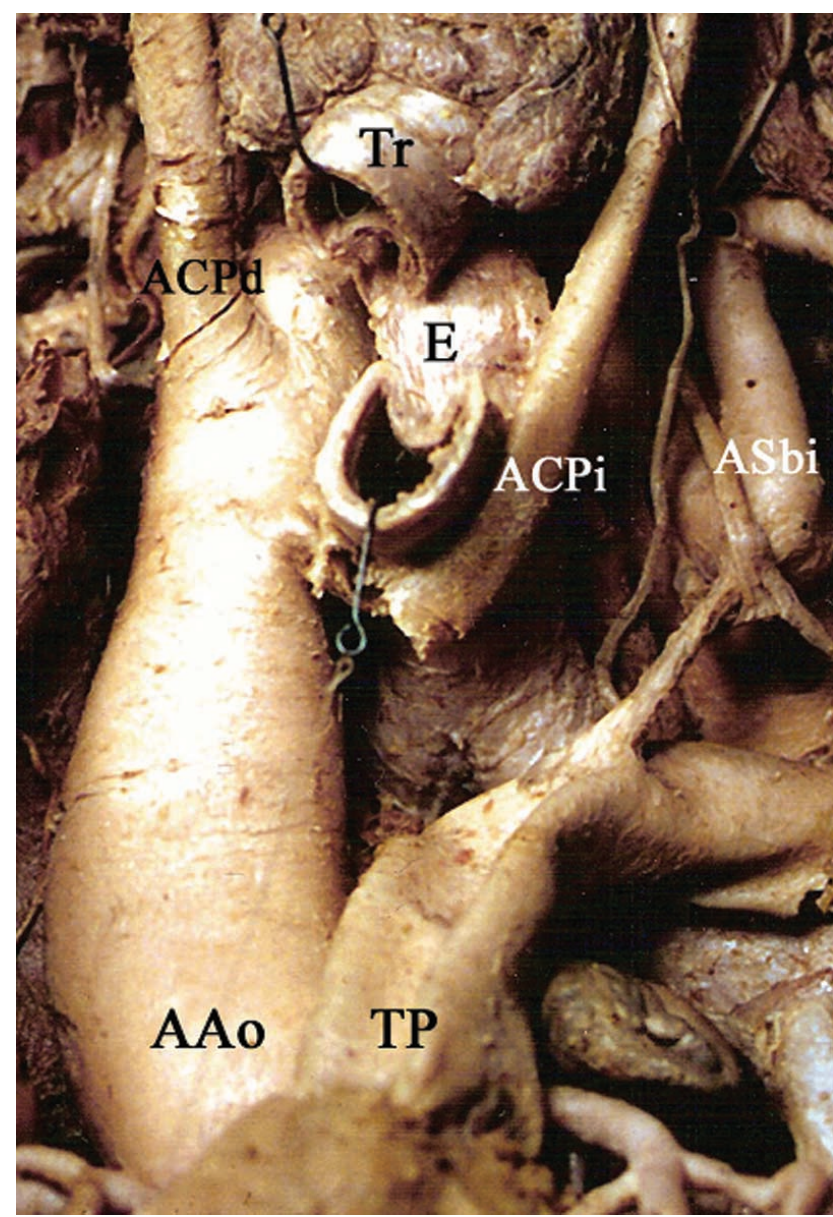

Fig. 5. Vista anterior con tráquea $(\operatorname{Tr})$ seccionada y rebatida hacia abajo y derecha. Se visualiza el arco aórtico (AAo) con las arterias carótidas comunes derecha (ACPd) e izquierda (ACPi) pretraqueal seccionada por la tracción que ejerce la tráquea al ser rebatida. Asimismo, el esófago (E), el tronco pulmonar (TP) y la arteria subclavia izquierda (ASbi).

\section{DISCUSIÓN}

Philip et al. (2001) hallaron que el origen pretraqueal de la carótida común izquierda tenía una incidencia del $62 \%$ en los casos de AARE y del $100 \%$ en los AARD. Hastreiter et al. (1966) identificaron que $9(7 \%)$ de 116 pacientes con AARED estaban asociados con tetralogía de Fallot. La incidencia de AARE con tetralogía de Fallot, varía de acuerdo a los reportes. Schneeweiss et al. (1984) encontraron tetralogía de Fallot en $5(5,5 \%)$ de sus series de 92 pacientes con AARED, pero Philip et al. no encontró que ninguno de los pacientes con AARED o AAREI estuviera asociado con tetralogía de Fallot. Steward et al. (1966) informaron que un caso estaba asociado con dextro-transposición de los grandes vasos y largo defecto del tabique ventricular, pero Philip et al. reportaron que 2 casos tenían defecto del tabique. Po- cos casos se asociaron con defecto del tabique interatrial, sólo Evans et al. (1950) reportaron tal anomalía y la asociaron íntimamente a las malformaciones halladas en pacientes con síndrome de Down (Tabla I). El hallazgo de una arteria subclavia retroesofágica en el curso del AARE es poco frecuente, pero altamente prevalente si la población en estudio con individuos con síndrome de Down, en los que se reportan arterias subclavias retroesofágicas hasta en el 37\% de los casos, acorde a investigaciones radiológicas de Goldstein et al. (1965).

La principal presión es ejercida sobre el esófago y la tráquea por el arco aórtico. En presencia de ligamento arterial o canal arterial o divertículo, pueden causar compresión adicional sobre tráquea y esófago. La sintomatología de esa compresión traqueo-esofágica son silbido, distress respiratorio, estridor, disfagia, dificultad en la alimentación, cianosis, vómitos o insuficiencia arterial (Kahn et al., 1977). Los casos sintomáticos con o sin anillo completo requieren intervención quirúrgica. Philip et al. reportaron que 2 casos (40\%) de los AARE derechos se presentaron con anillo vascular completo, mientras este anillo completo asciende

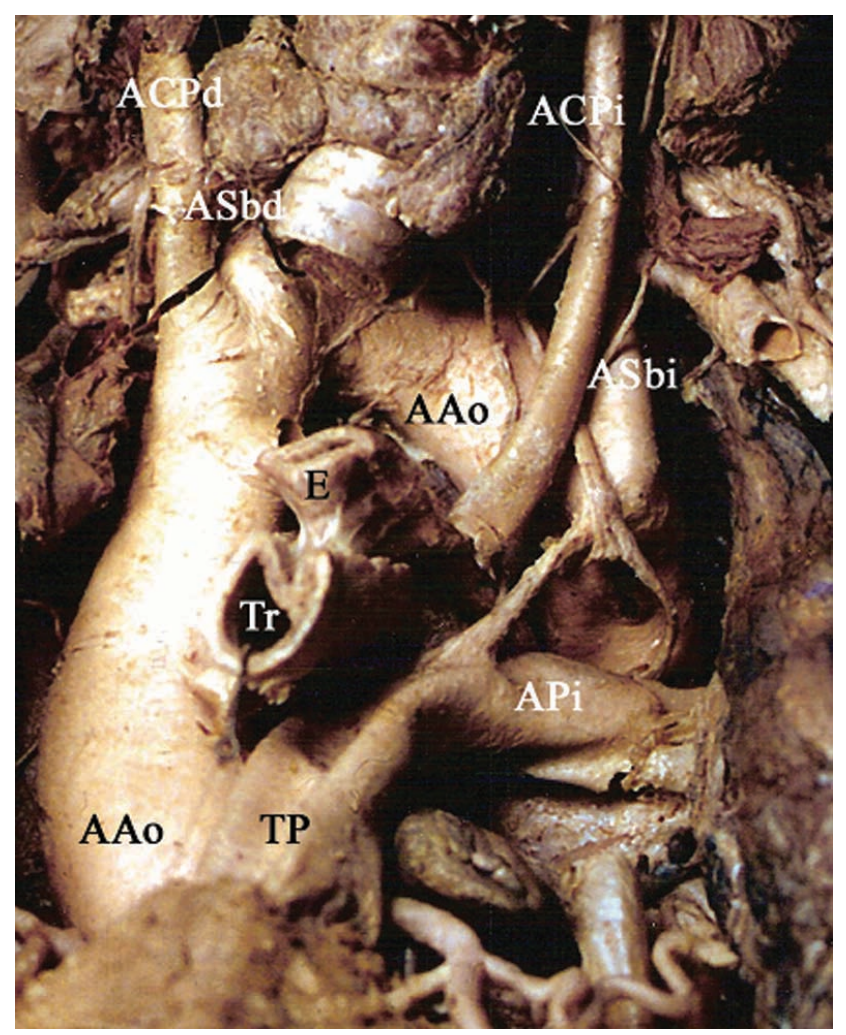

Fig. 6. Vista frontal con tráquea (Tr) y esófago (E) seccionados y rebatidos hacia abajo y derecha. Se visualizan todo el trayecto del arco aórtico retroesofágico (AAo), el origen de la arteria subclavia derecha (ASbd) en el arco aórtico en posición retroesofágica. Además, el tronco de la arteria pulmonar (TP), las arterias carótidas comunes derecha (ACPd) e izquierda (ACPi), la arteria subclavia izquierda (ASbi) y la arteria pulmonar izquierda (APi). 
al $66 \%$ en los casos de AARE izquierdo. La aorta forma un anillo vascular alrededor del esófago y de la tráquea, que se completa con un canal arterioso persistente o un ligamento arterioso. En este anillo vascular completo nunca se halló la participación de la arteria subclavia, sea ésta retroesofágica o no. La arteria subclavia retroesofágica no produce usualmente disfunción esofágica, pero conlleva defectos congénitos del corazón. Ningún reporte asocia a la arteria subclavia aberrante con el AARE izquierdo y son muy pocos los individuos en los que la arteria subclavia retroesofágica se presenta junto a un AARE derecho. La sensibilidad del diagnóstico temprano para el AARE por Rx tórax es de 37\%, mediante el esofagograma de $100 \%$ y con la angiografía de $60 \%$, pero la sensibilidad de la tomografía computada es del $100 \%$ en el trazado de ambos segmentos retroesofágicos y completo anillo vascular (Philip et al.).
La existencia de un AARE derecho es, a menudo, reconocida luego de un examen por imágenes (radiográfico, ecocardiográfico, tomográfico o por RMN) y, más aún, descubierta luego de una intervención quirúrgica cervicotorácica. En caso de disfagia, podría confirmarse por una opacificación del esófago en una endoscopía o mediante una seriada gastroduodenal. El síndrome de compresión traqueoesofágico con o sin enfermedad cardíaca congénita, aumenta la mortalidad, siendo el diagnóstico rápido de vital importancia. La AARE es diagnosticada mejor por tomografía computada con o sin reconstrucción 3D. La reconstrucción 3D o complementación con varios estudios de imágenes son útiles para conocer la posibilidad que esta variación vascular esté asociada a otra/s malformación/es, tales como arteria carótida pretraqueal, coartación aórtica o canal arterial persistente.

ALGIERI, R. D.; MAZZOGLIO Y NABAR, M. J. \& FERRANTE, M. S. Retroesophageal variation of the aortic arch. J. Morphol., 26(2):337-343, 2008.

SUMMARY: Classical literature does not mention the frequency of vascular thoracic changes. Nevertheless, these abnormalities are frequent, being able to exist without producing symptoms neither to require treatment. The retroesophageal aortic arch can be presented to the right (AARED) or left of the trachea and esophagus and cause symptoms of compression by means of a partial or complete vascular ring. It can be accompanied by abnormalities of the Subclavian Artery. In 1735 Hunauld reports the first case of aberrant Subclavian Artery, later, Hommel publishes a double aortic arch and in 1794 Bayford describes the "obstructive dysphagia lusoria". The aim was an anatomical description and a clinical and surgical correlate of a variation of the aortic arch such as an AARED. Cervicothoracical dissection of a adult male corpse with AARED found during the period 03/1998-03/2006, on 95 dissections formolized at $10 \%$. Results: In the AARED, the ascending aorta elapses on the right side of the esophagus and the trachea crossing over the mainstem bronchus, the arch crosses behind the esophagus and descends on the left side. Together with the arterial ligament, they form a vascular ring that wraps both the trachea and esophagus. Conclusions: The dissected corpse presents an AARED with a complete vascular ring and a right retroesophageal Subclavian Artery. These variations can be revealed by diagnostic imaging (esophagogram, angiography or computed tomography with or without 3-dimensional reconstruction) before the presence of a syndrome by esophagustracheal compression. The knowledge of these anomalies are extremely important for the surgeon in mediastinal approaches by the cervical way or in the urgency of a thoracic trauma.

KEY WORDS: Variations of the aortic arch; Retroesophageal aortic arch; Vascular ring; Retroesophageal subclavian Artery.

\section{REFERENCIAS}

Almear-García, V.; Terol, F. F.; Correa-Lacarcel, J.; Lloret, J.; Robles, J. \& del Campo, F. S. Retro-esophageal subclavian artery: a case report. Surg. Radiol. Anat., 24(34):231-4, 2002.

Arciniegas E. Vascular abnomalies compressing the trachea and esophagus. Pediatric surgery. Chicago, Year Book Medical Publishers Inc., 1979. pp649-52.

Blumenthal, S. \& Ravitch, M. M. Seminar on aortic vascular rings and other anomalies of the aortic arch. Pediatrics, 20(5, Part1):896-906, 1957.

Carlson, B. Embriología médica y biología del desarrollo. Madrid, Editorial Médica Panamericana, 2000.
Evans, W. The trend of cardiology. Overseas Postgrad. Med. J., 4(14):171, 1950.

Goldstein W. B. Aberrant right subclavian artery in mongolism. Am. J. Roentgenol. Radium Ther. Nucl. Med., 95:131-4, 1965.

Hastreiter, A. R.; D’Cruz, I. A.; Cantez, T.; Namin, E. P. \& Licata, R. Right-sided aorta. I. Occurrence of right aortic arch in various types of congenital heart disease. II. Right aortic arch, right descending aorta, and associated anomalies. Br. Heart J., 28(6):722-39, 1966.

Kahn, A.; Baran, D.; Spehl, M.; Dab, I. \& Blum, D. Congenital stridor in infancy. Clinical lessons derived 
from a survey of 31 instances. Clin. Pediatr. (Phila), 16(1):19-26, 1977.

Latarjet, M. \& Ruiz-Liard, A. Atlas de Anatomía. $2^{\mathrm{a}}$ ed. Buenos Aires, Panamericana, 2005.

Rouvière, H. \& Delmas, A. Anatomía Humana Descriptiva, Topográfica y Funcional. Tomo Tres. $10^{\mathrm{a}}$ ed. Barcelona, Masson, 1999.

Philip, S.; Chen, S. Y.; Wu, M. H.; Wang, J. K. \& Lue, H. C. Retroesophageal aortic arch: diagnostic and therapeutic implications of a rare vascular ring. Int. J. Cardiol., 79(23):133-41, 2001.

Schneeweiss, A.; Blieden, L.; Shem-Tov, A.; Deutsch, V. \& Neufeld, H. N. Retroesophageal right aortic arch. Pediatr. Cardiol., 5(3):191-5, 1984.

Stewart, J. R.; Kincaid, O. W. \& Titus, J. L. Right aortic arch: plain film diagnosis and significance. Am. J. Roentgenol. Radium Ther. Nucl. Med., 97(2):377-89, 1966.

Terracol, J. Les maladies de l'oesophage. Paris, Masson, 1938. p149.

Testut, L. \& Latarjet, A. Compendio de Anatomía Descriptiva. Barcelona, Salvat, 1981.

Williams, P. \& Warwick, R. Anatomía de Gray. Barcelona, Churchill Livingstone, 1992.
Dirección para correspondencia:

Rubén Daniel Algieri

Anatomía Humana

Facultad de Medicina.

Universidad de Buenos Aires

Paraguay $2055,4^{\circ}$ piso,

Sector Uriburu.

CP: 1121.

Buenos Aires

ARGENTINA

Email: mazzoglioynabar@hotmail.com

Recibido : 26-05-2007

Aceptado: 10-04-2008 
